\title{
Portal vein thrombosis: A concise review (Review)
}

\author{
RALUCA S. COSTACHE ${ }^{1,2}$, ANDREEA S. DRAGOMIRICĂ ${ }^{1}$, ELENA A. DUMITRAȘ ${ }^{1}$, \\ JINGA MARIANA ${ }^{1,2}$, ANA CĂRUNTU ${ }^{3}$, ANDRADA POPESCU ${ }^{1,2}$ and DANIEL O. COSTACHE ${ }^{4}$

\footnotetext{
${ }^{1}$ Department of Gastroenterology, 'Carol Davila' University Central Emergency Military Hospital, 010825 Bucharest;

${ }^{2} 5$ th Clinical Department, Gastroenterology and Internal Medicine Discipline,

'Carol Davila' University of Medicine and Pharmacy, 050474 Bucharest; ${ }^{3}$ Department of Maxillofacial Surgery;

${ }^{4}$ Department of Research, 'Carol Davila' University Central Emergency Military Hospital, 010825 Bucharest, Romania
}

Received January 20, 2021; Accepted February 19, 2021

DOI: $10.3892 /$ etm.2021.10191

\begin{abstract}
Portal vein thrombosis (PVT) is a frequent complication in cirrhotic patients, but it may also exist as a basic vascular condition even without any liver damage. Local and systemic factors play a significant role in the pathogenesis of PVT; yet, in practice, more than one factor may be identified. PVT can be considered a result of liver fibrosis and hepatic insufficiency. The JAK2 mutation has been accepted as a factor producing PVT. In general, the anticoagulants are recommended but this therapy should be used carefully in treating patients that associate coagulopathy or thrombocytopenia and esophageal varices. Acute PVT without bowel infarction has a good prognosis. In liver cirrhosis, the mortality due to hemorrhage is higher than in chronic PVT. Therefore, for the patients with PVT, the survival rate is decreased by $55 \%$ in two years, due to hepatic insufficiency. Regarding the treatment, LMWH (low molecular weight heparine) is the most utilized in patients with cirrhosis, non-malignancies, infections, or those who are awaiting a liver transplant. DOACs (direct-acting
\end{abstract}

Correspondence to: Mr. Daniel O. Costache, Department of Research, 'Carol Davila' University Central Emergency Military Hospital, Street Mircea Vulcănescu 88, 010825 Bucharest, Romania E-mail: daniel_costache@yahoo.com

Abbreviations: PVT, portal vein thrombosis; HCC, hepatocellular carcinoma; MPNs, myeloproliferative neoplasms; JAK2, JAK-2, Janus kinase 2; APA, antiphospholipid antibodies; CALR, calreticulin; PNH, paroxysmal nocturnal hemoglobinuria; MTHFR, methylenetetrahydrofolate reductase; BCS, Budd-Chiari syndrome; CT, computed tomography; MRI, magnetic resonance imaging; LMWH, low molecular weight heparin; LC, liver cirrhosis; VKA, vitamin $\mathrm{K}$ antagonist; DOAC, direct-acting oral anticoagulant; NASH, non-alcoholic steatohepatitis:, AT III (anti-thrombin III; CRP, $\mathrm{C}$ reactive protein; MELD score, Model for End-Stage Liver Disease score; VTE, venous thromboembolism; TIPS, transjugular intrahepatic portosystemic shunt; LT, liver transplantation)

Key words: portal vein thrombosis, Budd-Chiari, cirrhosis, portal cavernoma, anticoagulation, LMWH oral anticoagulants) may be used in the rest of the medical conditions, being safe and equal to LMWH.

\section{Contents}

1. Introduction

2. Epidemiology

3. Pathophysiology, risk factors, and genetic considerations

4. Clinical presentation

5. Diagnosis

6. Differential diagnosis

7. Interventions

8. Discussions

9. Conclusions

\section{Introduction}

Portal vein thrombosis (PVT) is a restriction or obstruction of the portal vein by a blood clot. Occlusion of the portal vein is a rare condition including the extra-hepatic segment and/or its subdivisions that appear simultaneously with mesenteric and/or splenic vein thrombosis (1). PVT often happens with liver cirrhosis but it can also be the result of another disorder, such as inherited thrombocytopenia, malignancies, abdominal infections, or bowel diseases (2). Physical examination, laboratory tests, and Eco-Doppler are useful to establish a rapid diagnosis and early treatment. Since PVT is a secondary disease, the guidelines adapt its treatment and duration to the underlying disease. The most studied were low molecular weight heparin (LMWH) and vitamin K antagonist. Although permeability occurs less frequently, anticoagulation may be used for several reasons: Anticoagulants are safe and advantageous, reduce the long-term complications of portal hypertension, and reduce or even eliminate thrombus. Direct-acting oral anticoagulants (DOACs) have not yet been studied and are recommended only in well-selected cases. In this review, we discussed risk factors, clinical cases, diagnostic methods, and currently most commonly used therapy tailored to the patient's profile, complications, and prognosis (3). Clinical consequences of PVT are constantly 
fluctuating, reliant on the dimension, extent, and measure of the thrombotic occlusion; and the parameters influence the clinical presentation, comorbidities, the appropriate treatment, complications, and prognosis (4).

\section{Epidemiology}

Portal vein thrombosis is a rare illness with a global incidence of $0.05-0.5 \%$ in post-mortem studies. The incidence differs, depending on the group of patients included (global population vs. patients with liver cirrhosis) and on the approach used to identify PVT condition (5). In liver cirrhotic patients that associate PVT, the incidence oscillates from 5 to $18 \%$ involving cases listed for liver transplant with an advanced degree of liver disease. Extra-hepatic PVT is valued to be around $5-10 \%$ of all cases of portal hypertension. Specifically, PVT frequency is higher for hepatic cirrhosis Child $\mathrm{B} / \mathrm{C}$ and is also associated with hepatocellular carcinoma (HCC), resulting in $1 \%$ of cases with a compensated disease, being elevated to $8-25 \%$ in transplant cases and higher, i.e., $40 \%$ in cases with diagnosis of HCC (6). In patients with advanced cirrhosis, non-alcoholic steatohepatitis (NASH) can be a distinct component of considerable thrombotic events, such as PVT (7-9). The prevalence varies depending on the diagnostic procedure: In angiography or surgical cases it is between 0.6 and $16 \%$, and in echography, it is $10-25 \%$ (10). In a study conducted on 1,234 patients with hepatic cirrhosis Child A or B, the overall incidence registered for PVT was: $4.6 \%$ in 1 year, $8.2 \%$ in 3 years, and $10.7 \%$ in 5 years. In 2016 in an Italian prospective study PRO-LIVER, realized on 753 cirrhotic patients the rate of ultrasound-detected PVT was $17 \%$ in cirrhosis, even though $43 \%$ of cases were subclinical. Since a higher number of abdominal imaging occurs in emergency rooms, the incidence of PVT has increased (11).

\section{Pathophysiology, risk factors, and genetic considerations}

The imbalance between procoagulant and anticoagulant factors induces hypercoagulability, which is responsible for venous thrombus formation. In profound venous thrombosis, the Virchow Triad can be applied: Venous stasis, endothelial dysfunction, and hypercoagulability status (12). (Tables I and II).

The following list of diseases has higher risks of developing PVT: Hepatocellular carcinoma (relative risk 124) (18), cholangiocarcinoma (relative risk 77), advanced hepatic cirrhosis (relative risk 15) (19), myeloproliferative disorders (relative risk 27) $(13,20)$. In advanced hepatic cirrhosis (Child B/C) the risk of developing PVT is high; either way, it is difficult to determine whether PVT worsens hepatic cirrhosis or advanced hepatic cirrhosis leads to PVT (21).

In the last years, it has been shown that patients with cirrhosis are susceptible to thrombus formation, even though anticoagulation and pro-coagulation factors are low. It has also been shown that low levels of protein $\mathrm{C}$, protein $\mathrm{S}$, and anti-thrombin III, as well as increased VIII endothelial factor and Virchow's triad can lead to a PVT condition that can complicate surgical procedures and liver transplantation on patients with hepatic cirrhosis Child B/C and elevated MELD Score (Model for End-Stage Liver Disease) (22).
The Factor V Leiden (FVL) G1691A and G20210A prothrombin mutations are considerably associated with PVT whether or not liver cirrhosis is present. Recently, the C677T mutation of methylenetetrahydrofolate reductase seems to be more common in association with prothrombotic disorders (such as PVT or Budd-Chiari syndrome), but in comparison with inherited thrombophilia is associated with a smaller incidence. In comparison with FVL, G20210A mutation is a moderate risk factor for venous obstruction $(\mathrm{OR}=2.8$ ), due to higher production of mRNA, which increases prothrombin amount, resulting in a thromboembolic condition (23).

Previous findings have shown that FVLG1691A, prothrombin G20210A mutation, and methylenetetrahydrofolate reductase (MTHFR) C677T are correlated with PVT in cirrhotic patients (increase progressively with Child-Pugh score) (24).

In an Italian study performed on non-malignant cirrhotic patients with acute PVT, the prevalence of both C677T mutation of MTHRF and prothrombin G20210A mutation was notably higher, due to a moderate level of hyperhomocysteinemia (a prothrombotic risk factor) resulting from C677T mutation of MTHFR activity (1).

Antiphospholipid antibodies (APA) are associated with a higher risk of developing PVT in patients with cirrhosis and $\mathrm{BCS}$. The prevalence in control studies is valued at approximately $5-15 \%$, but it is necessary to perform larger studies in this consideration because it is unknown if the hypercoagulability is connected to a synthetic malfunction or there is another genetic component or both.

Martinelli et al (25) mentioned increased factor VIII levels in cirrhotic patients with acute PVT, but substantial levels are observed in patients with thrombophilic conditions, but there are insufficient reports (3).

Myeloproliferative neoplasms (MPNs) such as polycythemia vera, essential thrombocythemia, and myelofibrosis, are associated with PVT, in the lack of malignancy of liver cirrhosis, and could be the first manifestation of disease. The detection of JAK2V16F (JAK-2, Janus kinase 2) mutation as a screening criterion of MPNs means a thrombotic condition should be considered (4).

Mutations of the gene codification for calreticulin (CALR) have been determined in $>80 \%$ of patients with primary myelofibrosis and $>65 \%$ of those with essential thrombocythemia with deprivation of the JAK2 mutation. Turon et al (26) established that CALR mutation may be identified in 3 of 4 patients of MPNs with PVT who had missed JAK2 mutation $(1,5)$.

Paroxysmal nocturnal hemoglobinuria (PNH) has been found in BCS patients (9-19\%) but it is also seen in patients with PVT (2\%). For diagnosis, it is necessary to determine CD55 and CD59 inadequate clones using flow cytometry. Antiphospholipid syndrome, pregnancy, and hormonal therapy are risk factors for PVT, however, other simultaneous etiological components should be identified. Bacteroides fragilis has been cited in patients with bacteremia, probably in consequence of anticardiolipin antibodies $(4,7)$.

Thus, a hematological screening especially for myeloproliferative neoplasms, inherited thrombophilia, and paroxysmal nocturnal hemoglobinuria is useful, albeit they are rare conditions; they are related to thrombosis at uncommon areas, especially in lack of liver cirrhosis or malignancy and certain family history $(5,11,27)$. 
Table I. Systemic causes of portal vein thrombosis.

\begin{tabular}{ll}
\hline Items & \multicolumn{1}{c}{ Results } \\
\hline Inherited thrombophilia & Factor V Leiden mutation; \\
& Prothrombin gene (G20210) mutation; \\
& Protein C, S, and antithrombin III (AT III) deficiency; \\
& Hyperhomocysteinemia \\
& Myeloproliferative disorders (JAK2 mutation, essential thrombocythemia, \\
Acquired thrombophilia & polycythemia vera, myelofibrosis); \\
& Antiphospholipid antibody syndrome/lupus anticoagulant; \\
& Paroxysmal nocturnal hemoglobinuria.
\end{tabular}

Table II. Localized causes of PVT.

Liver cirrhosis (28\% of PVT)

Malignancy (27-44\% of PVT)

Inflammatory conditions (10\% of PVT)

Injury to the portal vein

Abdominal infections

PVT, portal vein thrombosis.
Results

(Refs.)

Hepatocellular carcinoma;

Gastric and pancreatic adenocarcinoma;

Cholangiocarcinoma;

Lymphoma.

Appendicitis;

Cholecystitis;

Diverticulitis;

Pancreatitis;

Peptic ulcer perforation;

Inflammatory bowel disease.

Abdominal surgery (cholecystectomy, splenectomy,

gastric bypass surgery, colectomy);

Trauma, shunts, liver transplant.

Bacteriemia

$(10,16,17)$
There is one category of cirrhotic patients for which we can recommend laboratory tests for thrombophilia, those are the patients with stable hepatic cirrhosis who are developing PVT. It is shown that portal vein stasis leads to a decrease in portal vein velocity $(<15 \mathrm{~cm} / \mathrm{sec})$. Endothelial dysfunction is prone to infections that can decompensate the hepatic disorder and can produce PVT. PVT complications can be multiple but one of the most frequent are: Collateral circulation ( 2 weeks after the acute thrombosis), portal hypertension, variceal hemorrhage, ascites, and splenomegaly. Mesenteric infarction can be an acute form of presentation of PVT (28).

\section{Clinical presentation}

PVT depends on the primary disorder, and it is essential to establish whether it is associated with cirrhosis, non-malignant, malignant (abdominal solid tumors, lymphoma), or connected to thrombophilia, abdominal infections, sepsis because this guides the therapeutic approach. Clinical forms always depend on thrombus location, the degree of portal occlusion, and extension to the superior mesenteric vein, splenic vein, or both (29).
There are two categories of PVT presentation: Acute and chronic; even so, in practice, it may be difficult to discern between the types only from a physical examination. The usual presentation of acute PVT is symptomatic, including abdominal pain, nausea, and fever. Extension of mesenteric venous thrombosis associated with bowel ischemia may produce severe symptoms such as vomiting, diarrhea, rectal bleeding, and splenomegaly and in some cases even sepsis. If the occlusion is not promptly remediated, enteric perforation, peritonitis, shock, and multi-organ failure (MODS) may occur (30). Ascites is uncommon, it may be present before the progression of collateral circulation; while the majority of patients manifest splenomegaly. Without early treatment and management, mesenteric infarct may be the worst complication causing death (mortality at 6 weeks is $30 \%$ for cirrhotic patients) (31).

In a prospective study, Plessier et al (30) found that in non-cirrhotic cases, PVT was symptomatic, therefore $90 \%$ of patients exhibit abdominal pain, $53 \%$ of whom had a fever and more than $80 \%$ had high levels of CRP (C reactive protein) $(30,32)$. 
In advanced cirrhosis, PVT is a frequent complication, being crucial to early diagnosis due to decompensated portal hypertension that produces variceal hemorrhages (approximately 30\% of patients), affecting the pre- and post-transplant condition (33).

Acute variceal bleeding is the most frequent clinical manifestation of PVT. Usually, bleeding is well-tolerated and mortality is lower than in cirrhotic patients, perhaps as a result of liver preservation. Notably, 30-40\% of patients present gastric varices. In patients with cirrhosis it is more common to identify ectopic varices that appear in the duodenum, gallbladder bed, and the anorectal region; in these cases, portal hypertensive gastropathy is a rare condition (34).

Asymptomatic cases may appear due to spontaneous reabsorption of thrombus or it could be the manifestation of developed collateral circulation that leads to chronic PVT (35).

Portal vein thrombosis grading system (adapted after 36): Grade 1, $<50 \%$ PV thrombosed with or without extension to Superior Mesenteric Vein (SMV); Grade 2, >50\% PV thrombosed with or without extension to SMV; Grade 3, Complete thrombosis of PV and extension to proximal SMV; Grade 4, Complete thrombosis of PV and extension to proximal and distal SMV.

Classification PVT in cirrhosis (adapted after 36): i) Localization of PVT: Only trunk, only branch, both of branches, trunk and branches; ii) occlusion status: Occlusive/non-occlusive; iii) clinical presentation, recent form (Doppler examinations: Hyper-dense thrombus, PV dilatation), which can be asymptomatic/symptomatic, or chronic form (portal hypertension, collateral circulation) which can be asymptomatic/symptomatic (37).

Chronic PVT can be almost asymptomatic, excepting the appearance of varices, cutaneous collaterals, or ascites. It may be diagnosed when an ultrasound evaluation is performed for non-related problems, or investigations of portal hypertension (thrombocytopenia, jaundice, variceal bleeding, or splenomegaly). Portal cavernoma is a vascular neo-formation or an interconnection of collateral vessels bonding proximal and distal of the thrombus. Portal hypertension-related complications are the most common clinical expression of chronic PVT or portal cavernoma (38).

The risk of acute renal injury and sepsis is higher for elderly patients with advanced portal hypertension, chronic PVT-associated thrombocytopenia, and hypersplenism (4).

Another consequence of chronic PVT is bowel ischemia and portal biliopathy, which is defined as abnormalities of the extra, intra-hepatic bile ducts (the left one being more common) and gallbladder walls in the cases with portal cavernoma. It is diagnosed in $80-100 \%$ of cases, even though only a few are symptomatic, while $30 \%$ of these cases present jaundice, gall stone, cholangitis, and secondary biliary cirrhosis (39).

Zocco et al (40) studied the portal blood flow which can be slower $(<15 \mathrm{~cm} / \mathrm{sec})$ and it is considered a high predictive factor to develop PVT (28).

\section{Diagnosis}

Abdominal ultrasound is a reliable procedure with a high degree of sensitivity and specificity, with $60-100 \%$ used to diagnose PVT. In acute PVT, ultrasound shows hyperechoic material in the PV with secondary dilatation.
Doppler ultrasound is required to recognize a recently formed thrombus which may be anechoic. Doppler imaging measures portal blood flow which may be slower or absent $(<15 \mathrm{~cm} / \mathrm{sec})$. Portal cavernoma is suggestive of the chronic form of PVT, which commonly associates splenomegaly, splenic-portal collaterals, and portosystemic shunts. Color Doppler is considered the gold standard for diagnosis, especially in patients with liver cirrhosis with portal hypertension who are candidates for liver transplantation (LT) (sensitivity 80-100\%) (41).

Endoscopic ultrasonography has higher sensitivity and specificity comparable to eco-Doppler examination and it seems to be more precise than ultrasonography or CT scan in the estimation of portal invasion by abdominal tumors and even for better distinction between malignant, bland, or non-occlusive thrombus (42).

CT (computed tomography) and MRI (magnetic resonance imaging) furnish auxiliary information such as the status of adjacent organs, the evidence of possible complications (bowel infarction, bowel perforation), PVT etiology, vascular status, and hepatic disorders (HCC, liver abscess or other malignant tumors). On spin-echo MRI, the thrombus emerges isointense or hyperintense (if recent) on T1-weighted images and generally produces a more intense signal on T2 images. Contrast-enhanced MRI shows the flow direction and permeability of the portal venous system, being useful in the identification of the portal cavernoma $(42,43)$.

In patients with chronic PVT, an upper gastrointestinal endoscopy may suggest esophageal varices in $20-55 \%$ of cases, gastric varices in $40 \%$ of cases, and in limited patients, it may show the presence of antral-duodenal varices. The screening of varices is required 2-3 months after the acute PVT episode (42).

Patients with chronic PVT develop ectopic varices in the duodenum, anorectal region, and gallbladder bed. Magnetic resonance cholangiography is the most trusted technique used to assess portal biliopathy. In cases with no local cause identified it is important to eliminate a primary systemic disorder. When imaging performed is not conclusive, all patients should have a JAK2 analysis, factor V Leiden, prothrombin gene mutation, paroxysmal nocturnal hemoglobinuria, and lactate dehydrogenase tested. In PVT patients with ascites, the liver function tests are slightly abnormal. Alkaline phosphatase and gamma-glutamyl transpeptidase may be higher in portal biliopathy patients. Antiphospholipid syndrome and vasculitis analysis are also relevant (44).

\section{Differential diagnosis}

For acute abdominal conditions such as appendicitis, cholecystitis, cholangitis, pancreatitis, liver abscess, abdominal surgery, or trauma CT/MRI may be used to detect and indicate the differentiating features of acute thrombus, local intra-abdominal inflammatory condition, or both. There are several differences between cirrhosis and chronic PVT; in cirrhosis, we identify ascites, jaundice, hepatic encephalopathy, and liver functions are affected. Imaging in cirrhosis shows the irregular liver outline and also dilated portal vein, unlike the chronic form of PVT. Of note, for chronic PVT the conditions that liver cirrhosis presents do not apply (45). The 
splenomegaly is mild in cirrhosis and aggressive in chronic PVT. CT scan shows a non-enhancing filling defect in the background of the cirrhotic liver as differentiating features between PVT in cirrhosis and bland thrombosis. Due to malignant invasion, the CT scan shows filling defect with rim enhancement of vessel wall, interruption of the vessel wall, an expandable effect caused by the tumor mass, and the tumor itself (with or without cirrhotic liver) in PV invasion by HCC unlike PVT in cirrhosis. Other conditions that should be taken into consideration for the differential diagnosis are congenital hepatic fibrosis, Budd-Chiari Syndrome, sarcoidosis, schistosomiasis, primary biliary cirrhosis, and granulomatous hepatitis $(42,46)$.

\section{Interventions}

The treatment for portal vein thrombosis includes anticoagulation therapy, thrombolysis, thrombectomy, transjugular intrahepatic portosystemic shunt (TIPS). Current data suggest that anticoagulation is necessary, being the best therapeutic option in restoring vascular flow, aiming to prevent thrombus enlargement, prevent its reformation and also decrease the rate of complications (esophageal varices, mesenteric ischemia, or secondary peritonitis). Anticoagulation depends on the location of the obstruction, the extent of thrombosis, the degree of occlusion, the severity of the underlying disease and may also depend on the duration of the thrombotic episode (3).

Although there is no ideal anticoagulant, the use of LMWH is preferred. LMWH, such as enoxaparin, is associated with reduced markers of bacterial translocation and inflammation (22); dalteparin has a subcutaneous administration, is expensive and, in case of renal insufficiency the doses may be modified (contraindication at creatinine clearance $<10 \mathrm{ml} / \mathrm{min}$ ); vitamin $\mathrm{K}$ antagonists (VKA) such as Warfarin with oral administration INR $(2,3)$ should be carefully controlled; direct oral anticoagulants (DOACs) have to be avoided in renal dysfunction (contraindication at creatinine clearance $<30 \mathrm{ml} / \mathrm{min}$ ). However, none of the anticoagulants have been sufficiently investigated in different cases or cohorts. It seems to be a good therapeutic option in patients with secondary thrombocytopenia (induced by unfractionated heparin) (47). Indications for DOACs include, arrhythmias (14\%), PVT (61\%), Budd-Chiari syndrome (14\%), deep venous thrombosis (DVT) $(5 \%)$ and others $(5 \%)(48,49)$.

Concerning pharmacological methods, thrombolytic therapy is performed using urokinase or streptokinase with high success rates in non-cirrhotic non-malignant cases which develop symptomatic PVT, or for the patients in whom, despite the established antithrombotic treatment, the symptoms persist or worsen. As major bleeding is not to be neglected, this treatment should be reserved for severe, refractory forms. TIPS is indicated for patients with portal hypertension or for cirrhotic patients with variceal hemorrhage, prone to be transplanted, or with refractory ascites. It has an increased success rate $(>80 \%)$ in cirrhotic patients (approximately 500 cases in the literature) and non-cirrhotic patients with complete portal vein obstruction. It should be considered early in the management of PVT (50).

Thrombectomy is not routinely recommended because it is accompanied by increased morbidity and mortality, but thrombus aspiration during TIPS has been successfully tried (42).

For cirrhotic patients who develop acute PVT, there are various recommendations of guidelines and it is specified that in this category several factors must be considered. The guidelines (American Association for the Study of Liver Diseases) (12) recommend that anticoagulation needs to be adapted case by case (risk of variceal bleeding, thrombocytopenia). Anticoagulation is to be considered for at least 6 months at a therapeutic dose with LMWH/VKA (European Association for the Study of Liver) (51), or indefinite anticoagulation for patients with complications (intestinal ischemia) or patients with superior mesenteric vein thrombosis and/or splenic vein (54). TIPS is performed when there is severe portal hypertension or if PVT does not respond to the LMWH or thrombolysis may be practiced if the patient develops a mesenteric infarction (3).

In acute PVT in non-cirrhotic patients, the anticoagulation of all patients is recommended for $\geq 3$ months or long term. Such patients include those with uncorrectable thrombotic risk factors or symptomatic patients or those in whom the thrombus tends to expand at SMV (American Association for the Study of Liver Diseases). In the absence of contraindications, we can initiate therapy with LMWH or oral VKA for the long term (European Association for the Study of Liver). In patients with infection, antibiotics are needed to be promptly instituted (American Association for the Study of Liver Diseases) $(3,51)$.

In non-cirrhotic patients with malignancies, therapy should be for at least 6 months with LMWH or until the neoplasm resolves. In long-term therapy, we can choose between LMWH and VKA for patients with active cancer, metastases, or those who are being treated with chemotherapy. Thrombolysis is considered for patients who develop thrombosis, or a bowel infarct, despite the therapy with anticoagulants $(42,51)$.

PVT secondary to infections/surgeries are generally treated for 3-6 months, being able to choose between warfarin, LMWH, and DOACs (12).

In patients with PVT awaiting a liver transplant, the European Association for the Study of Liver (51) recommends considering the anticoagulation for patients with thrombus extension to SMV/SV or until the liver transplant has been completed or for patients who are candidates for liver transplant $(3,51)$.

In non-cirrhotic patients with chronic PVT, The American Association for the Study of Liver Diseases recommends anticoagulation for all patients with uncorrectable prothrombotic risk factors or myeloproliferative disorders. Permanent anticoagulation is recommended for patients with intestinal ischemia or severe prothrombotic disease or recurrent thrombosis $(3,51)$.

\section{Discussion}

In cirrhotic patients, Senzolo et al (52) reported in a prospective study that recanalization of the portal vein was successful in $94 \%$ of cases in patients treated with nadroparin in the first 6 months after acute presentation, compared with 5\% in which permeability was spontaneous for the untreated arm. The shortest time from the diagnosis and the establishment of the treatment ( $<6$ months) led to partial or total permeability. 
A single episode of cerebral hemorrhage was reported in the nadroparin arm.

Scheiner and colleagues (53) reported in a retrospective study that from 51 patients studied, those who received anticoagulants for $\geq 6$ months had a faster regression of thrombosis compared to those who received short-term anticoagulant. Given that LMWH and VKA were the most studied in the cirrhotic patient, no statistically significant differences were observed between reviews in terms of permeability at 6 months (3).

However, since LMWH is more commonly used than VKA, it has been observed that doses of $1.5 \mathrm{mg} / \mathrm{kg} /$ day have led to more frequent bleeding events compared to $1 \mathrm{mg} / \mathrm{kg}$ bid. In both studies, it appears that anticoagulants play a major role in permeability, and episodes of variceal hemorrhage were decreased in both studies. Nevertheless, we cannot generalize, since the studies are heterogeneous and have been performed on a small number of patients and yet current guidelines recommend therapeutic adaptation case by case (54).

DOACs are not sufficiently studied and are not recommended for the first line of treatment. A case report showed in a cirrhotic patient complete permeability of portal vein after 6 months of rivaroxaban treatment (55). Rivaroxaban, apixaban, and dabigatran has been studied in redacted portal vein thrombosis clinical trials and reports. Idarucizumab, a monoclonal antibody fragment that binds dabigatran, was accepted in 2015 by the USA Federal Drug Administration (FDA) to antagonize dabigatran anticoagulation (56).

Complications. The most frequent complications are bowel ischemia/infarction, variceal hemorrhage (which may be controlled using endoscopic banding and sclerotherapy in 95\% of acute hemorrhage cases and propranolol can be used after endoscopic therapy), hepatic encephalopathy, ascites, hepatic insufficiency (especially in cirrhosis), splenomegaly, and hypersplenism (42).

Mortality and prognosis. Mortality in patients with PVT is between 38 and $66 \%$ in 10 years, being secondary to the underlying disease. In the absence of liver disease, the risk of bleeding at 2 years is $0.25 \%$ compared to cirrhotic patients in whom the risk of bleeding is $>50 \%$.

The prognosis depends on the underlying disease and its long-term management, but overall, given the anticoagulant therapy the prognosis is good, $>70 \%$ of PVT patients survive $>10$ years after the acute event $(19,31,42)$.

\section{Conclusions}

Portal stasis, local and systemic risk factors such as inherited or acquired thrombophilia, coagulability conditions, abdominal surgery lead to endothelial damage and are decisive factors for PVT production. At the same time, PVT appearance is unexpected, even though we know that the principal condition for PVT formation is liver cirrhosis or malignancy. In most cases, we cannot neglect that genetic and acquired component in association with local risk factors (liver cirrhosis), together play a role in PVT occurrence (5). Previous findings have shown that G20210A prothrombin mutation is related to PVT in patients with LC (57). Nowadays, JAK2 mutation has been accepted as a producing factor for PVT and is potentially useful as a screening criterion of MNPs, in absence of LC or other disorders. Taking this into consideration, it is useful to perform larger studies regarding the long-term anticoagulation treatment for these patients. The portal flow velocity is reduced and constitutes a separate risk factor for patient prognosis; clinical guidelines recommend performing an Echo Doppler or contrast-enhanced CT scan which identifies portal cavernoma or a bland thrombus. Color Doppler is considered the gold standard for diagnosis, especially in patients with liver cirrhosis and portal hypertension determines who qualifies for liver transplantation. PVT is associated with critical hepatic diseases and contributes to deteriorating liver function and may complicate the acceptability of LT. Complete obstruction in patients awaiting liver transplantations has a negative prognosis in survival in comparison with patients where recanalization is complete, and thus the anticoagulation therapy is mandatory as quickly as possible to prevent bowel ischemia in the short-term and portal hypertension in the long-term. In absence of repermeabilization, portal cavernoma is a hemodynamic adjustment due to PVT and it appears as a balanced mechanism; the most significant complication is portal hypertension but anticoagulation therapy may be helpful. The studies are heterogeneous and were performed on a small number of patients. Nevertheless, current guidelines recommend therapeutic adaptation case by case, especially in cirrhotic patients. Anticoagulation remains the main therapy for acute PVT or chronic PVT (early anticoagulation has multiple benefits and it prevents severe complications with increasing survival rate) with uncorrectable prothrombotic risk factors. More classifications, and medical studies should be performed on a larger number of patients, with a focus on predictive markers, prognostic indicators, and severity scores. Currently, LMWH is the most utilized in patients with cirrhosis, non-malignancies, infections, or those awaiting a liver transplant. DOACs may be used in other medical conditions, being safe and equal to LMWH. TIPS can be regarded in the early treatment of PVT, with a success rate of $>80 \%$. The prognosis of PVT is dictated by the presence of baseline conditions (liver cirrhosis, MPNs, malignancy) and other risk factors.

\section{Acknowledgements}

Not applicable.

\section{Funding}

No funding was received.

\section{Availability of data and materials}

Not applicable.

\section{Authors' contributions}

RSC was involved in the conception and design of the work, as well as acquisition, analysis, and interpretation of data. ASD was responsible for the acquisition, analysis, and interpretation of data, as well as writing the first draft. EAD was involved in the acquisition, analysis, and interpretation of data for the 
study. JM was involved in the final approval of the version to be published. AC contributed to the acquisition, analysis, and interpretation of data for the study. AP was responsible for the acquisition, analysis, and interpretation of data for the work. DOC revised the article critically for important intellectual content. All authors read and approved the final manuscript.

\section{Ethics approval and consent to participate}

Not applicable.

\section{Patient consent for publication}

Not applicable.

\section{Competing interests}

The authors declare that they have no competing interests.

\section{References}

1. Samant H, Asafo-Agyei KO and Garfield K: Portal Vein Thrombosis. StatPearls Publishing LLC, 2021.

2. Iorga RA, Bratu OG, Marcu RD, Constantin T, Mischianu DLD, Socea B, Gaman MA and Diaconu CC: Venous thromboembolism in cancer patients: Still looking for answers. Exp Ther Med 18: 5026-5032, 2019.

3. Wu M, Schuster M and Tadros M: Update on management of portal vein thrombosis and the role of novel anticoagulants. J Clin Transl Hepatol 7: 154-164, 2019.

4. Ponziani FR, Zocco MA, Campanale C, Rinninella E, Tortora A, Di Maurizio L, Bombardieri G, De Cristofaro R, De Gaetano AM, Landolfi R and Gasbarrini A: Portal vein thrombosis: Insight into physiopathology, diagnosis, and treatment. World J Gastroenterol 16: 143-155, 2010.

5. Kumar A, Sharma P and Arora A: Review article: Portal vein obstruction-epidemiology, pathogenesis, natural history, prognosis and treatment. Aliment Pharmacol Ther 41: 276-292, 2015.

6. Manescu-Avram GV, Diaconu C, Voicu L, Jinga M, Ionita Radu F, Costache DO and Costache RS: Hepatocellular carcinoma-A review. Rom J Mil Med 120: 27-33, 2017.

7. Stine JG, Argo CK, Pelletier SJ, Maluf DG, Caldwell SH and Northup PG: Advanced non-alcoholic steatohepatitis cirrhosis: A high-risk population for pre-liver transplant portal vein thrombosis. World J Hepatol 9: 139-146, 2017.

8. Stine JG, Shah NL, Argo CK, Pelletier SJ, Caldwell SH and Northup PG: Increased risk of portal vein thrombosis in patients with cirrhosis due to nonalcoholic steatohepatitis. Liver Transpl 21: 1016-1021, 2015.

9. Ionită Radu F, Jinga M, Nută P, Costache RS, Bucurica S, Macadon B, Balaban V and Pătrăsescu M: Nonalcoholic fatty liver disease-an etiological approach. Rom J Mil Med 117: 33-39, 2014.

10. Okuda K, Ohnishi K, Kimura K, Matsutani S, Sumida M, Goto N, Musha H, Takashi M, Suzuki N and Shinagawa T, et al: Incidence of portal vein thrombosis in liver cirrhosis. An angiographic study in 708 patients. Gastroenterology 89: 279-286, 1985.

11. Violi F, Corazza RG, Caldwell SH, Perticone F, Gatta A, Angelico M, Farcomeni A, Masotti M, Napoleone L, Vestri A, et al: Portal vein thrombosis relevance on liver cirrhosis: Italian venous thrombotic events registry. Intern Emerg Med 11: 1059-1066, 2016

12. DeLeve LD, Valla DC, Garcia-Tsao G, Intagliata NM, Superina RA, Roberts LN, Lisman T and Valla DC: American Association for the Study Liver diseases. Vascular disorders of the liver. Hepatology 49: 1729-1764, 2009.

13. Dentali F, Galli M, Gianni M and Ageno W: Inherited thrombophilic abnormalities and risk of portal vein thrombosis: A meta-analysis. Thromb Haemost 99: 675-682, 2008.

14. Qi X, Chen H and Han G: Effect of antithrombin, protein C and protein Son portal vein thrombosis in liver cirrhosis: A meta-analysis. Am J Med Sci 346: 38-44, 2013.
15. Dentali F, Squizzato A, Brivio L, Appio L, Campiotti L, Crowther M, Grandi AM and Ageno W: JAK2V617F mutation for the early diagnosis of $\mathrm{Ph}$-myeloproliferative neoplasms in patients with venous thromboembolism: A meta-analysis. Blood 113: 5617-5623, 2009.

16. Ogren M, Bergqvist D, Bjorck M, Acosta S, Eriksson H and Sternby NH: Portal vein thrombosis: Prevalence, patient characteristics and lifetime risk: A population study based on 23,796 consecutive autopsies. World J Gastroenterol 12: 2115-2119, 2006.

17. Landman C, Nahon S, Cosnes J, Bouhnik Y, Brixi-Benmansour H, Bouguen G, Colombel JF, Savoye G, Coffin B, Abitbol V, et al: Portomesenteric vein thrombosis in patients with inflammatory bowel disease. Inflamm Bowel Dis 19: 582-589, 2013.

18. Al-Azzawi Y, Al-Abboodi Y, Fasullo M and Kheder J: Risk factors stratifications for portal venous thrombosis (PVT). J Liver 6: 208, 2007.

19. Fujiyama S, Saitoh S, Kawamura Y, Sezaki H, Hosaka T, Akuta N, Kobayashi M, Suzuki Y, Suzuki F, Arase Y, et al: Portal vein thrombosis in liver cirrhosis: Incidence, management, and outcome. BMC Gastroenterol 17: 112, 2017.

20. Popescu A, Gavrilă A, Nuță P, Costache RS, Jinga M, Bucurică S, Macadon B, Pătrășescu M and Ioniță Radu F: Case reporthepatocytolytic syndrome hiding mesenteric venous ischemia. Rom J Mil Med 118: 44-48, 2015.

21. Ponziani FR, Zocco MA, Garcovich M, D'Aversa F, Roccarina D and Gasbarrini A: What we should know about portal vein thrombosis in cirrhotic patients: A changing perspective. World J Gastroenterol 18: 5014-5020, 2012.

22. Francoz C, Valla D and Durand F: Portal vein thrombosis, cirrhosis, and liver transplantation. J Hepatol 57: 203-212, 2012.

23. Simsek E, Yesilyurt A, Pinarli F, Eyerci N and Ulus AT: Combined genetic mutations have remarkable effect on deep venous thrombosis and/or pulmonary embolism occurrence. Gene 536: 171-176, 2014.

24. Amitrano L, Guardascione MA, Brancaccio V, Margaglione M, Manguso F, Iannaccone L, Grandone E and Balzano A: Risk factors and clinical presentation of portal vein thrombosis in patients with liver cirrhosis. J Hepatol 40: 736-741, 2004.

25. Martinelli I, Primignani M, Aghemo A, Reati R, Bucciarelli P, Fabris F, Battaglioli T, Dell'Era A and Mannucci PM: High levels of factor VIII and risk of extra-hepatic portal vein obstruction. J Hepatol 50: 916-922, 2009.

26. Turon F, Cervantes F, Colomer D, Baiges A, Hernández-Gea V and GarciaPagán JC: Role of calreticulin mutations in the aetiological diagnosis of splanchnic vein thrombosis. J Hepatol 62: 72-74, 2015.

27. Draghici T, Negreanu L, Bratu OG, Stoian AP, Socea B, Neagu TP, Stanescu AMA, Manuc D and Diaconu CC: Paraneoplastic syndromes in digestive tumors: A review. Rom Biotechnol Lett 24: 813-819, 2019

28. Khoury T, Ayman AR, Cohen J, Daher S, Shmuel C and Mizrah M: The complex role of anticoagulation in cirrhosis: An updated review of where we are and where we are going. Digestion 93: 149-159, 2016.

29. Sarin SK, Philips CA, Kamath PS, Choudhury A, Maruyama H, Nery FG and Valla DC: Toward a comprehensive new classification of portal vein thrombosis in patients with cirrhosis. Gastroenterology 151: 574-577, 2016.

30. Plessier A, Darwish-Murad S, Hernandez-Guerra M, Consigny Y, Fabris F, Trebicka J, Heller J, Morard I, Lasser L, Langlet P, et al: Acute portal vein thrombosis unrelated to cirrhosis: A prospective multicenter follow-up study. Hepatology 51: 210-218, 2010.

31. Chawla YK and Bodh V: Portal vein thrombosis. J Clin Exp Hepatol 5: 22-40, 2015.

32. Amitrano L and Guardascione MA: Management of portal vein thrombosis in cirrhotic patients. Mediterr J Hematol Infect Dis 1: e2009014, 2009.

33. Cagin YF, Atayan Y, Erdogan MA, Dagtekin F and Colak C: Incidence and clinical presentation of portal vein thrombosis in cirrhotic patients. Hepatobiliary Pancreatic Dis Int 15: 499-503, 2016.

34. Faccia M, Ainora ME, Ponziani FR, Riccardi L, Garcovich M, Gasbarrini A, Pompili M and Zocco MA: Portal vein thrombosis in cirrhosis: Why a well-known complication is still matter of debate. World J Gastroenterol 25: 4437-4451, 2019.

35. De Gaetano AM, Lafortune M, Patriquin H, De Franco A, Aubin B and Paradis K: Cavernous transformation of the portal vein: Patterns of intrahepatic and splanchnic collateral circulation detected with Doppler sonography. AJR Am J Roentgenol 165: 1151-1155, 1995. 
36. Yerdel MA, Gunson B, Mirza D, Karayalçin K, Olliff S, Buckels J, Mayer D, McMaster P and Pirenne J: Portal vein thrombosis in adults undergoing liver transplantation: Risk factors, screening, management, and outcome. Transplantation 69: 1873-1881, 2000.

37. Iftimie G, Pantea Stoian A, Socea B, Motofei I, Marcu D, Costache RS and Diaconu C: Complications of systemic lupus erythematosus: A review. Rom J Mil Med 121: 9-15, 2018.

38. Young $\mathrm{K}$ and Wong R: Evaluation and management of acute and chronic portal vein thrombosis in patients with cirrhosis. Clin Liver Dis (Hoboken) 10: 152-156, 2017.

39. Sarin SK and Kumar A: Noncirrhotic portal hypertension. Clin Liver Dis 10: 627-651, 2006.

40. Zocco MA, Di Stasio E, De Cristofaro R, Novi M, Ainora ME, Ponziani F, Riccardi L, Lancellotti S, Santoliquido A, Flore R, et al: Thrombotic risk factors in patients with liver cirrhosis: Correlation with MELD scoring system and portal vein thrombosis development. J Hepatol 51: 682-689, 2009.

41. Margini $\mathrm{C}$ and Berzigotti A: Portal vein thrombosis: The role of imaging in the clinical setting. Dig Liver Dis 49: 113-120, 2017.

42. Khanna R and Sarin SK: Idiopathic portal hypertension and extrahepatic portal venous obstruction. Hepatol Int 12 (Suppl 1): S148-S167, 2018.

43. Diaconu C,Ciocîrlan M,Jinga M, Costache RS, Constantinescu G, Ilie $\mathrm{M}$ and Diculescu M: Ectopic pancreas mimicking gastrointestinal stromal tumor in the stomach fundus. Endoscopy 50 E186-E187, 2018.

44. Langabeer SE: CALR mutation analysis is not indicated in patients with splanchnic vein thrombosis without evidence of a myeloproliferative neoplasm: A micro-review. Ann Gastroenterol 29: 557-558, 2016.

45. Diaconu C, Ioniță Radu F, Jinga M, Nuță P, Bucurică S, Macadon B, Pătrășescu M, Popescu A, Balaban V, Voicu L, et al From liver biopsy to non-invasive markers in evaluating fibrosis in chronic liver disease. Rom J Mil Med 118: 5-12, 2015.

46. Vladut C, Ciocirlan M, Bilous D, Sandru V, Stan-Ilie M, Panic N, Becheanu G, Jinga M, Costache RS, Costache DO, et al: An overview on primary sclerosing cholangitis. J Clin Med 9: 754, 2020.

47. Joppa SA, Salciccioli J, Adamski J, Patel S, Wysokinski W, McBane R, Al-Saffar FR, Esser H and Shamoun F: A practical review of the emerging direct anticoagulants, laboratory monitoring, and reversal agents. J Clin Med 7: 29, 2018.
48. De Gottardi A, Trebicka J, Klinger C, Plessier A, Seijo S, Terziroli B, Magenta L, Semela D, Buscarini E, Langlet P, et al: Antithrombotic treatment with direct-acting oral anticoagulants in patients with splanchnic vein thrombosis and cirrhosis. Liver Int 37: 694-699, 2017.

49. Laslo CL, Bacalbasa N, Stanescu AMA, Carsote M, Bungau S, Rus M, Bratu OG and Diaconu CC: New oral anticoagulantspossible extension to other indications (Review). Exp Ther Med 20: 2401-2405, 2020

50. Qi X, Yang M, Fan D and Han G: Transjugular intrahepatic portosystemic shunt in the treatment of Budd-Chiari syndrome: A critical review of literatures. Scand J Gastroenterol 48: 771-784, 2013.

51. European Association for the Study of the Liver. Electronic address: easloffice@easloffice.eu: EASL clinical practice guidelines: Vascular diseases of the liver. J Hepatol 64: 179-202, 2016.

52. Senzolo M, Sartori TM, Rossetto V, Burra P, Cillo U, Boccagni P, Gasparini D, Miotto D, Simioni P, Tsochatzis E and A Burroughs K: Prospective evaluation of anticoagulation and transjugular intrahepatic portosystemic shunt for the management of portal vein thrombosis in cirrhosis. Liver Int 32: 919-927, 2012.

53. Scheiner B, Stammet PR, Pokorny S, Bucsics T, Schwabl P, Brichta A, Thaler J, Lampichler K, Ba-Ssalamah A, Ay C, et al: Anticoagulation in non-malignant portal vein thrombosis is safe and improves hepatic function. Wien Klin Wochenschr 130: 446-455, 2018.

54. Vega JA, Lee YR, McMahan D and Duong HQ: Monitoring enoxaparin with antifactor Xa levels in severe renal impairment. J Pharma Care Health Sys 3: 2, 2016.

55. Warkentin TE, Pai M and Linkins LA: Direct oral anticoagulants for treatment of HIT: Update of Hamilton experience and literature review. Blood 130: 1104-1113, 2017.

56. Connolly SJ, Milling TJ Jr, Eikelboom JW, Gibson CM, Curnutte JT, Gold A, Bronson MD, Lu G, Conley PB, Verhamme P, et al: Andexanet Alfa for acute major bleeding associated with factor Xa inhibitors. N Engl J Med 375: 1131-1141, 2016.

57. Smalberg JH, Arends LR, Valla DC, Kiladjian JJ, Janssen HL and Leebeek FW: Myeloproliferative neoplasms in Budd-Chiari syndrome and portal vein thrombosis: A meta-analysis. Blood 120: 4921-4928, 2012. 